TRANSACTIONS OF THE

AMERICAN MATHEMATICAL SOCIETY

Volume 357, Number 7, Pages 2829-2841

S 0002-9947(04)03626-8

Article electronically published on October 28, 2004

\title{
SADDLE SURFACES IN SINGULAR SPACES
}

\author{
DIMITRIOS E. KALIKAKIS
}

\begin{abstract}
The notion of a saddle surface is well known in Euclidean space. In this work we extend the idea of a saddle surface to geodesically connected metric spaces. We prove that any solution of the Dirichlet problem for the Sobolev energy in a nonpositively curved space is a saddle surface. Further, we show that the space of saddle surfaces in a nonpositively curved space is a complete space in the Fréchet distance. We also prove a compactness theorem for saddle surfaces in spaces of curvature bounded from above; in spaces of constant curvature we obtain a stronger result based on an isoperimetric inequality for a saddle surface. These results generalize difficult theorems of S.Z. Shefel ${ }^{\prime}$ on compactness of saddle surfaces in a Euclidean space.
\end{abstract}

\section{INTRODUCTION}

A surface in a Euclidean space is said to be a saddle surface if it is impossible to cut off a crust by any hyperplane. In this sense saddle surfaces are opposed to convex surfaces. In contrast to the theory of convex surfaces, the results in the theory of saddle surfaces are in many respects far from complete. Non-regular saddle surfaces in $\mathbb{E}^{n}$ were mainly studied by S.Z. Shefel' ([19], [20], [21]). Historically, saddle surfaces are connected with the Plateau problem in Euclidean spaces ([14).

In this work we extend the idea of a saddle surface to geodesically connected metric spaces and we study the class of saddle surfaces in spaces of curvature bounded from above in the sense of A.D. Aleksandrov. Spaces of bounded curvature inherit basic properties of Riemannian manifolds while they can have strong topological and metric singularities.

Saddle surfaces in a Euclidean space are usually defined by means of the operation of cutting off crusts by hyperplanes, or any other equivalent operation of hyperplanes ([21]). Therefore, a generalization of the classical definition to any metric space is not quite obvious. The definition we propose here for a saddle surface in a general geodesically connected metric space (Definition 3) makes use of the concept of convex hull and goes back to a property that a regular solution of the Plateau problem satisfies ([17, Lemma 7.1).

First, in Section 2, we recall what it means for a metric space to be of curvature bounded from above and we give the definition and some first examples of saddle surfaces in such spaces.

Received by the editors August 10, 2003 and, in revised form, December 2, 2003

2000 Mathematics Subject Classification. Primary 53C45, 53C43, 51M05.

Key words and phrases. Saddle surface, $\operatorname{CAT}(\kappa)$ space, curvature in the sense of A.D. Aleksandrov, isoperimetric inequality, compactness. 
In Section 3 we provide a source of examples of saddle surfaces in nonpositively curved spaces. We show that an energy minimizing surface in a complete nonpositively curved metric space is a saddle surface (Theorem 6). An energy minimizing surface is a Sobolev mapping with given trace which is stationary (among the mappings having the same trace) for the Sobolev energy. We note here that nonregular area minimizing surfaces are not in general saddle surfaces. There are many examples of non-Riemannian spaces which are nonpositively curved, e.g. trees, Euclidean buildings, Hilbert spaces, and other infinite dimensional symmetric spaces (4], [10]).

In Section 4 we show that in a nonpositively curved space the notion of a saddle surface is well defined for a general Fréchet surface (Proposition 12), and we prove that the space of saddle surfaces is complete in the Fréchet distance (Theorem 16).

A condition for compactness of a family of saddle surfaces in a compact space of bounded curvature, which assumes restrictions on area and lengths of bounding curves, is given in Section 5 (Theorem 17). In spaces of constant curvature we show that an isoperimetric inequality for the class of saddle surfaces (Theorem21) reduces compactness of a family of saddle surfaces to the compactness of the set of bounding curves (Theorem 23). These results generalize difficult theorems of S.Z. Shefel' on compactness of saddle surfaces in a Euclidean space ([21]).

\section{SAdDLE SURFACES IN METRIC SPACES OF CURVATURE BOUNDED FROM ABOVE BY $\kappa$}

2.1. Domain $\mathfrak{R}_{\kappa}$. A notion of curvature of metric spaces can be defined by comparing triangles in a metric space with the corresponding model triangles in the $\kappa$-plane with sides of the same length. The definition is due to A.D. Aleksandrov and the curvature is usually referred to as the curvature in the sense of A.D. Aleksandrov. Aleksandrov's spaces are a natural generalization of Riemannian manifolds but they are of a much more general nature. For more details, see [5].

The $n$-dimensional $\kappa$-space $\mathbb{S}_{\kappa}^{n}(\kappa$-plane for $n=2)$ is the hyperbolic space $\mathbb{H}_{\kappa}^{n}$ for $\kappa<0$, the Euclidean space $\mathbb{E}^{n}$ for $\kappa=0$, and the upper open hemisphere $\mathbb{S}_{+}^{n}\left(\kappa^{-1 / 2}\right)$ of $\mathbb{E}^{n+1}$ of radius $\kappa^{-1 / 2}$ with the induced metric, when $\kappa>0$. Every $\mathbb{S}_{\kappa}^{n}$ is a Riemannian simply connected manifold of constant sectional curvature $\kappa$ such that any pair of points can be joined by a unique geodesic segment - a curve of minimal length joining given pair of points. Notice that $\mathbb{S}_{\kappa}^{n}$ is a complete space only if $\kappa \leq 0$.

An $\mathfrak{R}_{\kappa}$ domain, abbreviated by $\mathfrak{R}_{\kappa}$, is a metric space satisfying the following axioms:

Axiom 1: Any two points in $\mathfrak{R}_{\kappa}$ can be joined by a geodesic segment.

Axiom 2: If $\kappa>0$, then the perimeter of each triangle in $\mathfrak{R}_{\kappa}$ is less than $\frac{2 \pi}{\sqrt{\kappa}}$.

Axiom 3: Each triangle in $\mathfrak{R}_{\kappa}$ has nonpositive $\kappa$-excess; that is, for the angles $\alpha, \beta, \gamma$ of a triangle $A B C$,

$$
\alpha+\beta+\gamma-\left(\alpha_{\kappa}+\beta_{\kappa}+\gamma_{\kappa}\right) \leq 0,
$$

where $\alpha_{\kappa}, \beta_{\kappa}, \gamma_{\kappa}$ are the corresponding angles of a triangle $A^{\kappa} B^{\kappa} C^{\kappa}$ on the $\kappa$-plane with sides of the same length as $A B C$

Another term for an $\mathfrak{R}_{\kappa}$ domain is a $C A T(\kappa)$ space. However, we will use Aleksandrov's original notation ([1]). It is evident that any $\kappa$-space is an $\mathfrak{R}_{\kappa}$ domain. 
A space of curvature bounded from above by $\kappa$ in the sense of A.D. Aleksandrov is a metric space with intrinsic metric, each point of which is contained in some neighborhood of the original space, which is an $\mathfrak{R}_{\kappa}$ domain.

We mention that any two points of $\mathfrak{R}_{\kappa}$ are joined by a unique geodesic segment, and any ball (of radius less than $\frac{\pi}{2 \sqrt{\kappa}}$, if $\kappa>0$ ) in an $\mathfrak{R}_{\kappa}$ domain is convex; that is, the geodesic segment joining any two points of the ball is still in the ball.

Finally, recall that a metric space with intrinsic metric is called geodesically connected if every pair of points can be joined by a geodesic segment.

2.2. Saddle surfaces. Let $M$ be a geodesically connected metric space with intrinsic metric. A surface $f$ in $M$ is any continuous mapping $f: D \rightarrow M$, where $D$ denotes the closed unit disk on the plane.

In the Euclidean space $\mathbb{E}^{n}$ we say that a hyperplane $P$ cuts off a crust from the surface $f$ if among the connected components of $f^{-1}(f(D) \backslash P)$ there is one with positive distance from the boundary of $D$. If $U$ is such a component, then the set $f(U)$ is called a crust. A surface $f$ in $\mathbb{E}^{n}$ is said to be a saddle surface if it is impossible to cut off a crust from it by any hyperplane.

We extend the idea of a saddle surface to any geodesically connected metric space by making use of the concept of convex hull. Let $A$ be a subset of $M$.

Definition 1. The convex hull of $A$, denoted by $\operatorname{conv}(A)$, is defined to be the closure of the union of all sets $G^{(n)}(A)$, with $G^{(0)}(A)=A, G^{(1)}(A)$ the union of all geodesic segments between points of $A$, and $G^{(n)}(A)=G^{(1)}\left(G^{(n-1)}(A)\right)$ for any $n>1$.

Remark 2. It follows directly from Definition 1 that if $x \in G^{(n)}(A)$ for some nonnegative integer $n$, then there exist finitely many points $a_{1}, \ldots, a_{m} \in A$ such that $x \in G^{(n)}\left(\left\{a_{1}, \ldots, a_{m}\right\}\right)$.

Notice that convex hull is always a closed set. In fact, in any $\mathfrak{R}_{\kappa}$ domain, $\operatorname{conv}(A)$ is the smallest closed convex subset containing $A$. Convex hull has special properties in nonpositively curved spaces.

We mention that if $\gamma$ is a Jordan plane curve, then $\mathbb{E}^{2} \backslash \gamma$ has exactly two connected components. We denote by int $\gamma$ the bounded component and by ext $\gamma$ the unbounded one.

Definition 3. A surface $f$ in a metric space $M$ is said to be a saddle surface if

$$
f(\operatorname{int} \gamma) \subset \operatorname{conv}(f(\gamma))
$$

for every Jordan curve $\gamma \subset D$ having positive distance from the unit circle.

Notice that saddle surfaces are, by definition, compact surfaces. Theorem 4 below shows the equivalence of Definition 3 with the classical one in the case of a Euclidean space.

Theorem 4 ([11]). If $f$ is a surface in $\mathbb{E}^{n}$, then the following are equivalent:

a) It is impossible to cut off a crust from $f$ by any hyperplane.

b) $f($ int $\gamma) \subset \operatorname{conv}(f(\gamma))$ for every Jordan curve $\gamma \subset D$ which has a positive distance from the unit circle.

Examples. 1) Any regular surface in $\mathbb{S}_{\kappa}^{3}$ with curvature not greater than $\kappa$ is a saddle surface. Notice that non-regular surfaces in $\mathbb{S}_{\kappa}^{3}$ with intrinsic curvature not greater than $\kappa$ are not necessarily saddle surfaces ([12]). 
2) Any ruled surface in an $\mathfrak{R}_{\kappa}$ domain is a saddle surface. The definition of a ruled surface in an $\mathfrak{R}_{\kappa}$ domain is the following. Let $\mathcal{L}$ be a closed curve in an $\mathfrak{R}_{\kappa}$ domain (whose length, when $\kappa>0$, is less than $\frac{2 \pi}{\sqrt{\kappa}}$ ), let $C$ be the unit circle, and let $f: C \rightarrow \mathfrak{R}_{\kappa}$ be a parametrization of $\mathcal{L}$. Let $O$ be an arbitrary point on $C$. Consider the surface whose parametrization $f$ is specified as follows: for any $X \in D$, lying on the line segment $O Y, Y \in C, f(X)$ lies on the geodesic segment $O^{\prime} Y^{\prime}$, where $O^{\prime}=f(O), Y^{\prime}=f(Y)$ and $O^{\prime} f(X): O^{\prime} Y^{\prime}=O X: O Y$. Because of the condition on the length of the curve $\mathcal{L}, O^{\prime} Y^{\prime}$ depends continuously on $Y^{\prime} \in \mathcal{L}$ and $f$ is a parametrized surface. In this case we say that we have a ruled surface with vertex $O^{\prime}$ spanned on $\mathcal{L}$. Based on this definition it is clear that if $O$ is a point on the unit circle $C$ and $f: D \rightarrow \mathfrak{R}_{\kappa}$ is a ruled surface with vertex $O^{\prime}=f(O)$ spanned on $f(C)$, then for any Jordan curve $\gamma$ in $D$ having positive distance from the unit circle, $f($ int $\gamma)$ is a subset of $G^{(1)}(f(\gamma))$. Therefore, $f($ int $\gamma) \subset \operatorname{conv}(f(\gamma))$.

$3)$ It is not difficult to see that saddle surfaces are preserved under the action of any continuous geodesic mapping. Since the inclusion mapping $i d: \mathbb{H}_{\kappa}^{n} \rightarrow \mathbb{E}^{n}$, where $\mathbb{H}_{\kappa}^{n}$ is interpreted by the Beltrami-Klein model of the hyperbolic space, and the central projection $\varphi: \mathbb{S}_{+}^{n}\left(\kappa^{-1 / 2}\right) \rightarrow \mathbb{E}^{n}$ are continuous geodesic mappings, we obtain the following characterization for saddle surfaces in $\mathbb{S}_{\kappa}^{n}$ : (i) a surface in $\mathbb{H}_{\kappa}^{n}$ is a saddle surface if and only if it is a saddle surfaces in $\mathbb{E}^{n}$, and (ii) a surface in $\mathbb{S}_{+}^{n}\left(\kappa^{-1 / 2}\right)$ is a saddle surface if and only if its image under the central projection is a saddle surface in $\mathbb{E}^{n}$.

\section{ENERGY MINIMIZING SURFACES}

An energy minimizing surface is an extremal of the energy functional with prescribed boundary condition. The energy of a mapping in a metric space was originally introduced by N. Korevaar and R. Shoen in [13] and independently by J. Jost in [9] in connection with the Dirichlet problem in nonpositively curved spaces. We recall what energy means in an $\mathfrak{R}_{0}$ domain and then we prove that every energy minimizing surface in a complete $\mathfrak{R}_{0}$ domain is a saddle surface (Theorem [6). In what follows $D$ is the unit closed disk in the Euclidean plane, $D_{\varepsilon}$ is the set of points in $D$ with dist $(x, \partial D) \geq \varepsilon$, and $D(x, \varepsilon)$ denotes the closed disk of radius $\varepsilon$ centered at $x$.

The class $L_{2}\left(D, \mathfrak{R}_{0}\right)$ is defined to be the set of all Borel-measurable surfaces $f: D \longrightarrow \mathfrak{R}_{0}$ having separable range such that $\int_{D} d^{2}(f(x), Q) d x<+\infty$, for some point $Q \in \mathfrak{R}_{0}$. If $\xi: D \longrightarrow[0,1]$ is a continuous function compactly supported in the interior of $D$ (notation: $\xi \in C_{0}(D)$ ), then the Sobolev class $W_{2}^{1}\left(D, \mathfrak{R}_{0}\right)$ is defined as the class of surfaces in $L_{2}\left(D, \Re_{0}\right)$ with finite energy $E_{f}$; where

$$
E_{f}=\sup _{\xi \in C_{0}(D) ; 0 \leq \xi \leq 1} \lim _{\varepsilon \rightarrow 0} \int_{x \in D_{\varepsilon}} \xi(x) \cdot\left(\int_{y \in B(x, \varepsilon)} \frac{d^{2}(f(x), f(y))}{\varepsilon^{4}} d y\right) d x .
$$

The set of $C^{\infty}$-smooth functions $h: D \longrightarrow \mathbb{R}$ which are compactly supported in the interior of $D$ is denoted by $C_{0}^{\infty}(D)$. We define the class $\hat{W}_{2}^{1}(D, \mathbb{R})$ as the completion of the set $C_{0}^{\infty}(D)$ with respect to the $W_{2}^{1}$-norm. Let $f, g \in W_{2}^{1}\left(D, \mathfrak{R}_{0}\right)$. We say that the surfaces $f$ and $g$ have the same trace if the function $h(x)=d(f(x), g(x))$ is in the class $\hat{W}_{p}^{1}(D, \mathbb{R})$. Let $\varphi \in W_{2}^{1}\left(D, \Re_{0}\right)$. Denote by $W_{2}^{1}\left(D, \mathfrak{R}_{0}, \varphi\right)$ the set of 
surfaces $f \in W_{2}^{1}\left(D, \Re_{0}\right)$ such that $f$ has the same trace as $\varphi$. Let

$$
\mathfrak{D}(\varphi)=\inf _{f \in W_{2}^{1}\left(D, \Re_{0}, \varphi\right)} E_{f} .
$$

A surface in the class $W_{2}^{1}\left(D, \mathfrak{R}_{0}, \varphi\right)$ whose energy is equal to $\mathfrak{D}(\varphi)$ is called an energy minimizing surface. The solution of the Dirichlet problem in nonpositively curved spaces is provided by the following theorem.

Theorem 5 (9], 13]). Let $\varphi \in W_{2}^{1}\left(D, \mathfrak{R}_{0}\right)$ be a surface in a complete $\mathfrak{R}_{0}$ domain. Then there exists a unique surface $f \in W_{2}^{1}\left(D, \mathfrak{R}_{0}, \varphi\right)$ with energy $E_{f}$ equal to $\mathfrak{D}(\varphi)$. Moreover, $f$ is locally Lipschitz continuous in the interior of $D$.

Theorem [6] is a source of examples for saddle surfaces in nonpositively curved spaces.

Theorem 6. Every energy minimizing surface in a complete $\mathfrak{R}_{0}$ domain is a saddle surface.

The proof of Theorem 6 is based on Lemma 11 which is a refinement of the Definition 3, and on a lemma of H. Busemann and W. Feller. Lemma 11 enable us to handle the definition of saddle surface using Jordan plane curves of zero Lebesgue measure. We need some extra terminology and lemmas.

Definition 7 (Similar points). Let $\left\{x_{1}, \ldots, x_{k}\right\}$ and $\left\{y_{1}, \ldots, y_{k}\right\}$ be two finite ordered sets in some geodesically connected metric space $M$. Two points $x \in\left\{x_{1}, \ldots, x_{k}\right\}$ and $y \in\left\{y_{1}, \ldots, y_{k}\right\}$ are called similar if there is some $i_{0} \in\{1, \ldots, k\}$ such that $x=x_{i_{0}}$ and $y=y_{i_{0}}$. Generally, two points $x \in G^{(n)}\left(\left\{x_{1}, \ldots, x_{k}\right\}\right)$ and $y \in$ $G^{(n)}\left(\left\{y_{1}, \ldots, y_{k}\right\}\right)$ are called similar if there are points $x^{\prime}, x^{\prime \prime} \in G^{(n-1)}\left(\left\{x_{1}, \ldots, x_{k}\right\}\right)$, and $y^{\prime}, y^{\prime \prime} \in G^{(n-1)}\left(\left\{y_{1}, \ldots, y_{k}\right\}\right)$ and some number $t=t(x, y, n) \in[0,1]$ such that: (i) $x$ lies on the geodesic segment joining $x^{\prime}$ to $x^{\prime \prime}$, and $y$ lies on the geodesic segment joining $y^{\prime}$ to $y^{\prime \prime}$, (ii) $x^{\prime} x=t \cdot x^{\prime} x^{\prime \prime}$, and $y^{\prime} y=t \cdot y^{\prime} y^{\prime \prime}$, and (iii) $x^{\prime}$ is similar to $y^{\prime}$, and $x^{\prime \prime}$ is similar to $y^{\prime \prime}$.

Lemma 8. Let $\left\{x_{1}, \ldots, x_{k}\right\}$ and $\left\{y_{1}, \ldots, y_{k}\right\}$ be two finite ordered sets of points in an $\mathfrak{R}_{0}$ domain, and let $x \in G^{(n)}\left(\left\{x_{1}, \ldots, x_{k}\right\}\right)$ for some positive integer $n$. If $y \in G^{(n)}\left(\left\{y_{1}, \ldots, y_{k}\right\}\right)$ is a point similar to $x$, then the following inequality holds:

$$
d(x, y) \leq \max _{i=1, \ldots, k} d\left(x_{i}, y_{i}\right) .
$$

Proof. We need the following lemma due to Yu.G. Reshetnyak.

Lemma 9 (18]). Let $\{P, Q, R, S\}$ be a quadruple of distinct points in an $\mathfrak{R}_{0}$ domain. For $t \in[0,1]$, define $P_{t} \in P S, Q_{t} \in Q R$ by

$$
P P_{t}=t \cdot P S \text { and } Q Q_{t}=t \cdot Q R \text {. }
$$

Then for any $t \in[0,1]$ the following inequality holds: $\left(P_{t} Q_{t}\right)^{2} \leq(1-t) \cdot P Q^{2}+t$. $R S^{2}-t(1-t) \cdot(S P-Q R)^{2}$.

If $n=0$, then (1) holds as an equality. Suppose that (1) holds for the nonnegative integer $n-1$. Let $x \in G^{(n)}\left(\left\{x_{1}, \ldots, x_{k}\right\}\right)$ and $y \in G^{(n)}\left(\left\{y_{1}, \ldots, y_{k}\right\}\right)$ such that $x$ is similar to $y$. Then there are points $x^{\prime}, x^{\prime \prime} \in G^{(n-1)}\left(\left\{x_{1}, \ldots, x_{k}\right\}\right)$, and $y^{\prime}, y^{\prime \prime} \in$ $G^{(n-1)}\left(\left\{y_{1}, \ldots, y_{k}\right\}\right)$ and some number $t \in[0,1]$ satisfying the conditions (i), (ii), 
and (iii) of Definition [7. Then, by Lemma 9,

$$
\begin{aligned}
d(x, y) & \leq \sqrt{(1-t) \cdot d^{2}\left(x^{\prime}, y^{\prime}\right)+t \cdot d^{2}\left(x^{\prime \prime}, y^{\prime \prime}\right)} \leq \max \left\{d\left(x^{\prime}, y^{\prime}\right), d\left(x^{\prime \prime}, y^{\prime \prime}\right)\right\} \\
& \leq \max _{i=1, \ldots, k} d\left(x_{i}, y_{i}\right),
\end{aligned}
$$

where the last inequality is based on the inductive assumption.

Lemma 10. Let $\left\{\gamma_{n}: n \in \mathbb{N}\right\}$, and let $\gamma$ be parametrized Jordan curves in an $\mathfrak{R}_{0}$ domain such that $\lim _{n \rightarrow \infty} \gamma_{n}=\gamma$ uniformly. Then for any sequence $\left\{x_{n}: n \in \mathbb{N}\right\}$ with $x_{n} \in \operatorname{conv}\left(\gamma_{n}\right)$ there is a sequence $\left\{y_{n}: n \in \mathbb{N}\right\} \subseteq \operatorname{conv}(\gamma)$ such that

$$
d\left(x_{n}, y_{n}\right) \leq \alpha_{n}, \text { for all } n \in \mathbb{N}
$$

with $\lim _{n \rightarrow \infty} \alpha_{n}=0$.

Proof. Let $n$ be fixed and $x_{n} \in \operatorname{conv}\left(\gamma_{n}\right)$. Suppose $x_{n} \in G^{(k)}\left(\gamma_{n}\right)$, for some $k \in \mathbb{N}$. Then, by Remark 2, there are points $t_{1}, t_{2}, \ldots, t_{m}$ on the unit circle $S^{1}$ such that $x_{n} \in \operatorname{conv}\left\{\gamma_{n}\left(t_{1}\right), \ldots, \gamma_{n}\left(t_{m}\right)\right\}$. Consider the points $\gamma\left(t_{1}\right), \ldots, \gamma\left(t_{m}\right)$ on the curve $\gamma$ and define the point $y_{n} \in \operatorname{conv}\left\{\gamma\left(t_{1}\right), \ldots, \gamma\left(t_{m}\right)\right\}$ to be similar to the point $x_{n}$, according to Definition 7 . Then, by Lemma 8 ,

$$
d\left(x_{n}, y_{n}\right) \leq \max _{i=1 \ldots m} d\left(\gamma_{n}\left(t_{i}\right), \gamma\left(t_{i}\right)\right) \leq \sup _{t \in S^{1}} d\left(\gamma_{n}(t), \gamma(t)\right) .
$$

Taking $\alpha_{n}=\sup _{t \in S^{1}} d\left(\gamma_{n}(t), \gamma(t)\right)$, we have $d\left(x_{n}, y_{n}\right) \leq \alpha_{n}$ for all $n \in \mathbb{N}$ and $\lim _{n \rightarrow \infty} \alpha_{n}=0$.

Suppose $x_{n}$ belongs in the closure of the union of all $G^{(k)}\left(\gamma_{n}\right), k \in \mathbb{N}$. Then choose a point $z_{n}$ in some $G^{(k)}\left(\gamma_{n}\right)$ so that $d\left(z_{n}, x_{n}\right) \leq \sup _{t \in S^{1}} d\left(\gamma_{n}(t), \gamma(t)\right)$. For this $z_{n}$ there is a $y_{n} \in \operatorname{conv}(\gamma)$ such that $d\left(z_{n}, y_{n}\right) \leq \sup _{t \in S^{1}} d\left(\gamma_{n}(t), \gamma(t)\right)$. An application of the triangle inequality completes the proof.

Lemma 11. Let $f: D \longrightarrow \mathfrak{R}_{0}$ be a surface in an $\mathfrak{R}_{0}$ domain. The following are equivalent:

a) $f$ is a saddle surface,

b) $f($ int $\gamma) \subseteq \operatorname{conv}(f(\gamma))$ for every Jordan polygonal curve $\gamma$ in $D$ with positive distance from the boundary of $D$.

Proof. The implication a) $\Rightarrow$ b) is trivial. Let $\gamma$ be a Jordan curve in $D$ with $\operatorname{dist}(\gamma, \partial D)>0$, and $\left\{\gamma_{n}: n \in \mathbb{N}\right\}$ be a sequence of Jordan polygonal curves in $D$ with $\operatorname{dist}\left(\gamma_{n}, \partial D\right)>0$ such that $\lim _{n \rightarrow \infty} \gamma_{n}=\gamma$ uniformly. Then $f\left(\gamma_{n}\right)$ uniformly converges to $f(\gamma)$ since $f$ is uniformly continuous on the compact set $D$. Suppose, contrary to the claim, that $f($ int $\gamma)$ is not a subset of $\operatorname{conv}(f(\gamma))$. Then there is a point $s \in$ int $\gamma$ with $x \equiv f(s) \notin \operatorname{conv}(f(\gamma))$. Let $d>0$ be the distance of $x$ from convf $(\gamma)$. Since $s \in$ int $\gamma$ and $\lim _{n \rightarrow \infty} \gamma_{n}=\gamma$ uniformly, there exists a positive integer $N=N(s)=N(x)$ such that $s \in$ int $\gamma_{n}$ for all $n \geq N$. Therefore, $x \equiv f(s) \in f\left(\right.$ int $\left.\gamma_{n}\right)$ for all $n \geq N$, and hence, $x \in \operatorname{conv}\left(f\left(\gamma_{n}\right)\right)$ for all $n \geq N$, since $f\left(\right.$ int $\left.\gamma_{n}\right) \subseteq \operatorname{conv}\left(f\left(\gamma_{n}\right)\right)$. By Lemma [10, choose a sequence $\left\{y_{n}: n \in \mathbb{N}\right\} \subseteq \operatorname{conv}(\gamma)$ so that $d\left(x, y_{n}\right) \leq \alpha_{n}$ for all $n \geq N$ with $\lim _{n \rightarrow \infty} \alpha_{n}=0$. Then $\operatorname{dist}(x, \operatorname{conv} f(\gamma))=0$. This is a contradiction.

Proof of Theorem [6. Let $f$ be an energy minimizing surface in a complete $\mathfrak{R}_{0}$ domain. Then there is a mapping $\varphi \in W_{2}^{1}\left(D, \mathfrak{R}_{0}\right)$ such that $f$ is the unique solution of the Dirichlet problem

$$
\mathfrak{D}(\varphi)=\inf _{f \in W_{2}^{1}\left(D, \Re_{0}, \varphi\right)} E_{f}
$$


Suppose that $f$ is not a saddle surface. Then, by Lemma 11, there exists a Jordan, polygonal curve $\gamma$ in $D$ such that $\operatorname{dist}(\gamma, \partial D)>0$ and $f($ int $\gamma)$ is not a subset of $\operatorname{conv}(f(\gamma))$. Consider the nearest point projection

$$
\pi: \mathfrak{R}_{0} \longrightarrow \operatorname{conv}(f(\gamma)) \text {. }
$$

This is a well-defined mapping, since conv $(f(\gamma))$ is a closed, convex set in a metric space of curvature bounded above by zero and, by a lemma of $\mathrm{H}$. Busemann and W. Feller ([15]), it is a non-expanding mapping; that is, $d(\pi(P), \pi(Q)) \leq d(P, Q)$, for all $P, Q \in \mathfrak{R}_{0}$. Define $g: D \longrightarrow \mathfrak{R}_{0}$ by

$$
g(u)=\left\{\begin{array}{c}
(\pi \circ f)(u), \text { if } u \in(\text { int } \gamma) \cup \gamma, \\
f(u), \text { if } u \in \text { ext } \gamma .
\end{array}\right.
$$

Obviously, $g($ int $\gamma) \subseteq \operatorname{conv}(g(\gamma))$ and $g \in W_{2}^{1}\left(D, \Re_{0}, \varphi\right)$. We claim that $E_{g} \leq$ $E_{f}$. This inequality leads to a contradiction since, by uniqueness, $g=f$ which contradicts the hypothesis that $f($ int $\gamma)$ is not a subset of conv $(f(\gamma))$. The energy of $g$ is given by the formula

$$
E_{g}=\sup _{\xi \in C_{0}(D) ; 0 \leq \xi \leq 1} \lim _{\varepsilon \rightarrow 0} \int_{x \in D_{\varepsilon}} \xi(x) \cdot\left(\int_{y \in B(x, \varepsilon)} \frac{d^{2}(g(x), g(y))}{\varepsilon^{4}} d y\right) d x .
$$

For any sufficiently small $\varepsilon>0$, we define $A_{\varepsilon}=\left\{z \in D_{\varepsilon}: \operatorname{dist}(z, \gamma) \leq \varepsilon\right\}$. Then for any $\varepsilon>0, D_{\varepsilon}$ is decomposed into the three disjoint components int $\gamma \backslash A_{\varepsilon}, A_{\varepsilon}$, and ext $\gamma \cap D_{\varepsilon} \backslash A_{\varepsilon}$. By definition of $g$ and $A_{\varepsilon}$ we have: (i) $d(g(x), g(y)) \leq d(f(x), f(y))$, if $x \in$ int $\backslash A_{\varepsilon}$, and $y \in B(x, \varepsilon)$, and (ii) $d(g(x), g(y))=d(f(x), f(y))$, if $x \in$ $\operatorname{ext} \gamma \cap D_{\varepsilon} \backslash A_{\varepsilon}$, and $y \in B(x, \varepsilon)$. On the other hand,

$$
\lim _{\varepsilon \rightarrow 0} \int_{x \in A_{\varepsilon}} \xi(x) \cdot\left(\int_{y \in B(x, \varepsilon)} \frac{d^{2}(g(x), g(y))}{\varepsilon^{4}} d y\right) d x=0,
$$

since $\lim _{\varepsilon \rightarrow 0} \mu\left(A_{\varepsilon}\right)=\mu(\gamma)=0$, where $\mu$ is the Lebesgue measure in $\mathbb{E}^{2}$. Therefore, $E_{g} \leq E_{f}$. The proof is complete.

\section{Completeness of the SPACE OF SADDle SURFACES}

4.1. Fréchet equivalence. Given any two surfaces $f_{1}: D \rightarrow M$ and $f_{2}: D \rightarrow$ $M$ we say that $f_{1}$ and $f_{2}$ are Fréchet equivalent (notation $f_{1} \sim f_{2}$ ) if given any positive number $\varepsilon$, there exists a homeomorphism $h_{\varepsilon}: D \rightarrow D$ such that $d\left(f_{1}(x), f_{2}\left(h_{\varepsilon}(x)\right)\right)<\varepsilon$, for all $x \in D$. It is known that $\sim$ is an equivalence relation in the collection of surfaces. In addition, $f_{1}(D)=f_{2}(D)$ whenever $f_{1} \sim f_{2}$. If $f$ is a surface the set of all surfaces $f^{\prime}$ with $f \sim f^{\prime}$ is an equivalence class, denoted by $[f]$, and is called a Fréchet surface; each element of $[f]$ is called a parametrization of the Fréchet surface. From the geometric point of view it would be desirable to find properties of saddle surfaces which are invariant under Fréchet equivalence. First we prove that Definition 3 of a saddle surface makes sense for Fréchet surfaces in nonpositively curved spaces.

Proposition 12. If $f_{1}: D \rightarrow \mathfrak{R}_{0}$ and $f_{2}: D \rightarrow \mathfrak{R}_{0}$ are Fréchet equivalent surfaces in an $\mathfrak{R}_{0}$ domain, and $f_{2}$ is a saddle surface, then $f_{1}$ is a saddle surface. 
We need the following technical lemma.

Lemma 13. Let $K$ be a subset of an $\mathfrak{R}_{0}$ domain, and let $\left\{z_{n}: n \in \mathbb{N}\right\}$ be a sequence of points in $\mathfrak{R}_{0}$ such that $z_{n} \in$ conv $\bigcup_{y \in K} B\left(y, \frac{1}{n}\right)$ for all $n \in \mathbb{N}$ and $\lim _{n \rightarrow \infty} z_{n}=z$ for some $z \in \Re_{0}$. Then $z \in \operatorname{conv}(K)$.

Proof. The convex hull is by definition a closed set, so it suffices to prove that for any $z_{n} \in \operatorname{conv} \bigcup_{y \in K} B\left(y, \frac{1}{n}\right)$ there is a point $x_{n} \in \operatorname{conv}(K)$ such that $d\left(z_{n}, x_{n}\right) \leq \frac{2}{n}$. Let $n$ be fixed. Suppose $z_{n} \in G^{(k)}\left(\bigcup_{y \in K} B\left(y, \frac{1}{n}\right)\right)$, for some positive integer $k=k(n)$. Then, by Remark[2, there are $y_{1}, \ldots, y_{m} \in K$ and $a_{1} \in B\left(y_{1}, \frac{1}{n}\right), \ldots, a_{m} \in B\left(y_{m}, \frac{1}{n}\right)$ such that $z_{n} \in G^{(k)}\left(\left\{a_{1}, \ldots, a_{m}\right\}\right)$. Choose the point $x_{n} \in G^{(k)}\left(\left\{y_{1}, \ldots, y_{m}\right\}\right) \subset$ $\operatorname{conv}(K)$ to be similar to the point $z_{n}$ according to Definition [7] Then, by Lemma 8 we have $d\left(z_{n}, x_{n}\right) \leq \max _{i=1, \ldots, m} d\left(a_{i}, y_{i}\right) \leq \frac{1}{n}$.

Suppose that $z_{n}$ belongs to the closure of the union of all $G^{(k)}\left(\bigcup_{y \in K} B\left(y, \frac{1}{n}\right)\right)$, $k \in \mathbb{N}$. Choose a point $y_{n}$ in some $G^{(k)}\left(\bigcup_{y \in K} B\left(y, \frac{1}{n}\right)\right)$ such that $d\left(y_{n}, z_{n}\right) \leq \frac{1}{n}$, and a point $x_{n} \in \operatorname{conv}(K)$ such that $d\left(y_{n}, x_{n}\right) \leq \frac{1}{n}$. The triangle inequality implies the desired inequality.

Proof of Proposition 12, Let $\varepsilon>0$. Then there exists a homeomorphism $h_{\varepsilon}: D \rightarrow$ $D$ such that

$$
d\left(f_{1}(x), f_{2}\left(h_{\varepsilon}(x)\right)\right)<\varepsilon, \text { for all } x \in D .
$$

Let $\gamma$ be a Jordan curve in $D$ having positive distance from the unit circle, and $x \in$ int $\gamma$. Then $h_{\varepsilon}(x) \in h_{\varepsilon}(i n t \gamma)=\operatorname{int}\left(h_{\varepsilon}(\gamma)\right)$, and therefore,

$$
f_{2}\left(h_{\varepsilon}(x)\right) \in f_{2}\left(\operatorname{int}\left(h_{\varepsilon}(\gamma)\right)\right) \subset \operatorname{conv} f_{2}\left(h_{\varepsilon}(\gamma)\right)
$$

since $f_{2}$ is a saddle surface. Set $z_{\varepsilon} \equiv f_{2}\left(h_{\varepsilon}(x)\right)$. Then (2) and (31) imply $f_{1}(x) \in$ $B\left(z_{\varepsilon}, \varepsilon\right)$, with $z_{\varepsilon} \in \operatorname{conv} f_{2}\left(h_{\varepsilon}(\gamma)\right)$. But $f_{2}\left(h_{\varepsilon}(\gamma)\right) \subset \bigcup_{y \in f_{1}(\gamma)} B(y, \varepsilon)$, by (2) . So

$$
f_{1}(x) \in B\left(z_{\varepsilon}, \varepsilon\right), \text { with } z_{\varepsilon} \in \operatorname{conv} \bigcup_{y \in f_{1}(\gamma)} B(y, \varepsilon) .
$$

Now taking $\varepsilon=1 / n$, we can assume, without loss of generality, that the sequence $z_{1 / n} \equiv f_{2}\left(h_{1 / n}(x)\right) \in f_{2}(D)$ converges to some point $z$, since $f_{2}(D)$ is a compact set. Taking limits as $n \rightarrow \infty$ in (4), and using Lemma 13, we have $f_{1}(x)=z$ and $z \in \operatorname{conv} f_{1}(\gamma)$. Thus, $f_{1}($ int $\gamma) \subset \operatorname{conv} f_{1}(\gamma)$. The proof is complete.

4.2. The space of saddle surfaces. Let $f_{1}: D \rightarrow M$ and $f_{2}: D \rightarrow M$ be two surfaces. The Fréchet distance $\mathfrak{F}\left(f_{1}, f_{2}\right)$ of $f_{1}$ and $f_{2}$ is defined to be

$$
\mathfrak{F}\left(f_{1}, f_{2}\right)=\inf \sup \left\{d\left(f_{1}(x), f_{2}(h(x))\right): x \in D\right\},
$$

where the infimum is taken over all possible homeomorphisms $h: D \rightarrow D$. It is known that $\mathfrak{F}\left(f_{1}, f_{2}\right)=\mathfrak{F}\left(f_{1}^{\prime}, f_{2}^{\prime}\right)$ whenever $f_{1} \sim f_{1}^{\prime}$ and $f_{2} \sim f_{2}^{\prime}$, therefore $\mathfrak{F}\left(\left[f_{1}\right],\left[f_{2}\right]\right) \equiv \mathfrak{F}\left(f_{1}, f_{2}\right)$ is a well-defined relation. $\mathfrak{F}$ defines a metric on the set of Fréchet surfaces which is called the Fréchet metric. 
Theorem 14. Saddle surfaces in an $\mathfrak{R}_{0}$ domain form a closed set in the space of surfaces with the Fréchet metric.

We need the following elementary lemma.

Lemma 15. Let $\left\{f_{n}\right\}_{n \in N}$ be a sequence of surfaces in a metric space $M$ which converges to a surface $f$, in the Fréchet sense. Then every sequence of the form $\left\{f_{n}\left(x_{n}\right)\right\}_{n \in \mathbb{N}}$, with $x_{n} \in D$, has a convergent subsequence. The limit point lies in the graph of $f$.

Proof. Let $n$ be fixed. Then there exists a positive integer $N=N(n)$ such that if $m \geq N(n)$, then $\mathfrak{F}\left(f_{m}, f\right)<\frac{1}{n}$. So, there exists a homeomorphism $h_{n, m}$ : $D \rightarrow D$ such that $d\left(f_{m}(x), f\left(h_{n, m}(x)\right)<\frac{1}{n}\right.$, for all $x \in D$ and $m \geq N(n)$. Let $\left\{l_{n}: n \in \mathbb{N}\right\}$ be an increasing sequence of positive integers such that $l_{n} \geq N(n)$. Then $d\left(f_{l_{n}}(x), f\left(h_{n, l_{n}}(x)\right)<\frac{1}{n}\right.$, for all $x \in D$. The sequence $\left\{h_{n, l_{n}}\left(x_{l_{n}}\right)\right\}_{n \in \mathbb{N}}$ has a convergent subsequence $\left\{h_{k_{n}, l_{k_{n}}}\left(x_{l_{k_{n}}}\right)\right\}_{n \in \mathbb{N}}$ since $D$ is compact. Say $\lim _{n \rightarrow \infty}$ $h_{k_{n}, l_{k_{n}}}\left(x_{l_{k_{n}}}\right)=a$. By the triangle inequality, we have

$$
\begin{aligned}
d\left(f_{l_{k_{n}}}\left(x_{l_{k_{n}}}\right), f(a)\right) & \leq d\left(f_{l_{k_{n}}}\left(x_{l_{k_{n}}}\right), f\left(h_{k_{n}, l_{k_{n}}}\left(x_{l_{k_{n}}}\right)\right)+d\left(f\left(h_{k_{n}, l_{k_{n}}}\left(x_{l_{k_{n}}}\right), f(a)\right)\right.\right. \\
& \leq \frac{1}{k_{n}}+d\left(f\left(h_{k_{n}, l_{k_{n}}}\left(x_{l_{k_{n}}}\right), f(a)\right)\right.
\end{aligned}
$$

which approaches zero, as $n \rightarrow \infty$, since $f$ is continuous.

Proof of Theorem 14. Let $\left\{f_{n}\right\}_{n \in \mathbb{N}}$ be a sequence of saddle surfaces in an $\mathfrak{R}_{0}$ domain converging to a surface $f$ in the Fréchet sense, and let $\varepsilon>0$. Then there exists a positive integer $n_{0}=n_{0}(\varepsilon)$ such that $\mathfrak{F}\left(f_{n}, f\right)<\varepsilon$, if $n \geq n_{0}$. Hence, there exists a homeomorphism $h_{\varepsilon, n}: D \rightarrow D$ such that

$$
d\left(f(x), f_{n}\left(h_{\varepsilon, n}(x)\right)\right)<\varepsilon \text { for all } x \in D .
$$

Let $\gamma$ be a Jordan curve in $D$, having positive distance from the unit circle, and $x \in$ int $\gamma$. Then $h_{\varepsilon, n}(x) \in h_{\varepsilon, n}(\operatorname{int} \gamma)=\operatorname{int}\left(h_{\varepsilon, n}(\gamma)\right)$, and therefore,

$$
f_{n}\left(h_{\varepsilon, n}(x)\right) \in f_{n}\left(\operatorname{int}\left(h_{\varepsilon, n}(\gamma)\right)\right) \subset \operatorname{conv} f_{n}\left(h_{\varepsilon, n}(\gamma)\right)
$$

since $f_{n}$ is a saddle surface. Set $z_{\varepsilon, n} \equiv f_{n}\left(h_{\varepsilon, n}(x)\right)$. Then (5) and (6) imply $f(x) \in$ $B\left(z_{\varepsilon, n}, \varepsilon\right)$, with $z_{\varepsilon, n} \in \operatorname{conv} f_{n}\left(h_{\varepsilon, n}(\gamma)\right)$. But $f_{n}\left(h_{\varepsilon, n}(\gamma)\right) \subset \bigcup B(y, \varepsilon)$, by (彁). Hence, $f(x) \in B\left(z_{\varepsilon, n}, \varepsilon\right)$ with $z_{\varepsilon, n} \in \operatorname{conv} \bigcup_{y \in f(\gamma)} B(y, \varepsilon)$. Taking $\varepsilon \rightarrow 0$, and choosing a convergent subsequence of $z_{\varepsilon, n} \equiv f_{n}\left(h_{\varepsilon, n}(x)\right)$, insured by Lemma 15, we have, by Lemma 13, that $f(x) \in \operatorname{conv} f(\gamma)$. The fact that $x$ is an arbitrary point in int $\gamma$ yields $f($ int $\gamma) \subset \operatorname{conv} f(\gamma)$. The proof is complete.

It is known that the space of Fréchet surfaces is a complete metric space relative to the Fréchet distance, so we obtain the following theorem.

Theorem 16. The space of saddle surfaces in an $\mathfrak{R}_{0}$ domain is complete with respect to the Fréchet metric.

\section{Compactness theorem}

The area of a surface in a general metric space was originally introduced by I.G. Nikolaev in connection with the Plateau Problem ([15]). The following theorem provides a condition for compactness of a family of saddle surfaces in a compact space of bounded curvature which assumes restrictions on area and lengths of bounding curves. 
Theorem 17. Let $\left\{\left[f_{\alpha}\right]\right\}_{\alpha \in A}$ be a family of saddle surfaces in a compact $\mathfrak{R}_{\kappa}$ domain with Jordan bounding curves. If the areas and the lengths of the bounding curves of the surfaces of the given family are uniformly bounded, then $\left\{\left[f_{\alpha}\right]\right\}_{\alpha \in A}$ is a relatively compact subset of the space of surfaces with respect to the Fréchet metric.

To prove Theorem 17 we need the following lemmas.

Lemma $18([8])$. Let $\mathcal{L}$ be a Jordan curve in a metric space. Then, for any $\varepsilon>0$, there exists $\tau(\varepsilon)>0$ such that if $Q, Q^{\prime}$ is a pair of points on $\mathcal{L}$ at a distance not exceeding $\tau(\varepsilon)$, the diameter of one of the two arcs of $\mathcal{L}$ determined by the points $Q$ and $Q^{\prime}$ does not exceed $\varepsilon$.

A corollary of Lemma 18 is the following lemma.

Lemma 19 (15]). Let there be given a sequence $\left\{\mathcal{L}_{n}\right\}_{n \in \mathbb{N}}$ of Jordan curves in a metric space which converges, in the Fréchet sense, to a Jordan curve $\mathcal{L}$. Then, for any $\varepsilon>0$, there exists a $\tau(\varepsilon)>0$ such that if the distance between two points on any of the curves $\mathcal{L}_{n}$ and $\mathcal{L}$ is less than $\tau(\varepsilon)$, the diameter of one of the two arcs into which these points divide the chosen curve is less than $\varepsilon$.

Fix a point $\mathbf{x}$ in the closed unit disk $D$ of the Euclidean plane. Denote by $S_{r}$ the arc of the circle centered at $\mathbf{x}$ of radius $r$ which is contained in $D$. Let $f: D \rightarrow \mathfrak{R}_{\kappa}$ be a parametrized surface. Denote by $\ell_{r}$ the length of the curve $f\left(S_{r}\right)$ in $\mathfrak{R}_{\kappa}$. We say that the parametrized surface $f$ satisfies Courant's condition relative to a constant $C>0$ if for each $x \in D$ and $\delta \in(0,1)$ there exists a $\rho \in[\delta, \sqrt{\delta}]$ such that

$$
\ell_{\rho} \leq \frac{C}{\sqrt{\ln \frac{1}{\delta}}}
$$

Lemma 20 ([15]). If the areas of a sequence $\left\{f_{n}\right\}_{n \in \mathbb{N}}$ of parametrized surfaces in a complete, locally compact $\mathfrak{R}_{\kappa}$ domain are uniformly bounded, then each $f_{n}, n=$ $1,2, \ldots$, satisfies the Courant's condition relative to a constant $C>0$ independent of $n$.

Proof of Theorem 17. It suffices to prove that each sequence $\left\{\left[f_{n}\right]\right\}_{n \in \mathbb{N}} \subset\left\{\left[f_{\alpha}\right]\right\}_{\alpha \in A}$ is a relative compact set, or equivalently, by the Arzelá-Ascoli Theorem that: for every $\varepsilon>0$ there exists $\delta>0$ such that $d\left(f_{n}(x), f_{n}(y)\right)<\varepsilon$, for all $n \in \mathbb{N}$, provided $x, y \in D$ and $|x-y|<\delta . d$ is the metric of $\mathfrak{R}_{\kappa}$, and $f_{n}$ is any parametrization of $\left[f_{n}\right]$.

Let $\varepsilon>0$. For each $n \in \mathbb{N}$ let us fix a parametrization $f_{n}$ for the surface $\left[f_{n}\right]$. Since the lengths of the bounding curves of $\left\{f_{n}\right\}_{n \in \mathbb{N}}$ are uniformly bounded then, by Hilbert's Theorem, the sequence of these bounding curves is a relatively compact set. So it has a convergent subsequence in the Fréchet sense. For the sake of simplicity let us assume that the sequence of bounding curves converges. Let $\tau(\varepsilon)$ be the uniform constant ensured by Lemma 19. Also, since the areas of the sequence $\left\{f_{n}\right\}_{n \in \mathbb{N}}$ are uniformly bounded, then by Lemma 20, each $f_{n}, n=1,2, \ldots$, satisfies Courant's condition relative to a uniform constant $C>0$. Choose $\delta>0$ such that

$$
\frac{C}{\sqrt{\ln \frac{1}{\delta}}}<\min \left\{\frac{\varepsilon}{3}, \tau\left(\frac{\varepsilon}{3}\right)\right\} .
$$


Let $x, y \in D$ with $|x-y|<\delta$. Put $z=\frac{x+y}{2}$. Then by Courant's condition, there exists a $\rho \in[\delta, \sqrt{\delta}]$ such that

$$
\ell_{\rho, n} \leq \frac{C}{\sqrt{\ln \frac{1}{\delta}}}, \text { for all } n \in \mathbb{N},
$$

with $\ell_{\rho, n}$ the length of $f_{n}\left(S_{\rho}\right)$, where $S_{\rho}$ is the arc of the circle centered at $\mathbf{z}$ of radius $\rho$ which is contained in $D$. Let $U$ be the intersection of $D$ with the closed disk centered at $\mathbf{z}$ of radius $\rho$, and $\gamma$ its boundary.

Suppose $U \subset D$. Then $S_{\rho}=\gamma$ and therefore $\ell\left(f_{n}(\gamma)\right)<\frac{\varepsilon}{3}$. So $f_{n}(\gamma)$ is contained in a closed ball in $\Re_{\kappa}$ of radius $\frac{\varepsilon}{3}$. But $f_{n}$ is a saddle surface and in any $\mathfrak{R}_{\kappa}$ domain closed balls, of sufficiently small radius, are convex sets. Hence,

$$
f_{n}(U) \subset \operatorname{conv}\left(f_{n}(\gamma)\right) \subset \text { ball of radius } \frac{\varepsilon}{3} .
$$

On the other hand, $\delta \leq \rho \leq \sqrt{\delta}$, so $|x-y|<\delta$ yields $x, y \in U$. Thus, $d\left(f_{n}(x), f_{n}(y)\right)$ $<\varepsilon$, for all $n \in \mathbb{N}$.

In the case when $U$ is not a subset of $D$, then the boundary of $U$ consists of two curves: the $\operatorname{arc} S_{\rho}$ and an arc, say $\gamma_{1}$, of the unit circle. These two arcs intersect at two points $p, q$ of the unit circle. Let $P_{n}=f_{n}(p)$ and $Q_{n}=f_{n}(q)$. Then $P_{n}, Q_{n}$ are joined by the curve $f_{n}\left(S_{\rho}\right)$ so by (7) and (8)

$$
d\left(P_{n}, Q_{n}\right)<\tau\left(\frac{\varepsilon}{3}\right) \quad \text { for all } n \in \mathbb{N} .
$$

The points $P_{n}, Q_{n}$ divide the boundary curve of $f_{n}$ into two arcs: $f_{n}\left(\gamma_{1}\right)$ and $f_{n}\left(\gamma_{2}\right)$, where $\gamma_{2}$ is the complement of $\gamma_{1}$ in the unit circle. By Lemma 19 the diameter of one of them is less than $\frac{\varepsilon}{3}$. Suppose that the diameter of $f_{n}\left(\gamma_{1}\right)$ is less than $\frac{\varepsilon}{3}$. Then the diameter of $f_{n}\left(\gamma_{1}\right) \cup f_{n}\left(S_{\rho}\right)$ is not greater than $\frac{2 \varepsilon}{3}$ so, since $f_{n}$ is a saddle surface,

$$
f_{n}(U) \subset \operatorname{conv}\left(f_{n}\left(\gamma_{1}\right) \cup f_{n}\left(S_{\rho}\right)\right) \subset \text { ball of radius } \frac{\varepsilon}{3} .
$$

Therefore, if $|x-y|<\delta$, then $x, y \in U$ and hence, $d\left(f_{n}(x), f_{n}(y)\right)<\varepsilon$ for all $n \in \mathbb{N}$. If the diameter of $f_{n}\left(\gamma_{2}\right)$ is less than $\frac{\varepsilon}{3}$, then instead of the parametrization $f_{n}$ we consider the parametrization $f_{n} \circ \varphi_{n}$, where $\varphi_{n}$ is a homeomorphism of $D$ which maps $\gamma_{1}$ onto $\gamma_{2}$ and the region $U$ onto the region bounded by $\gamma_{2}$ and $S_{\rho}$. The proof is complete.

In spaces of constant curvature saddle surfaces satisfy the following isoperimetric inequality.

Theorem 21. If a saddle surface in a compact subset $K$ of $\mathbb{S}_{\kappa}^{n}$ is bounded by a curve of length $\ell$, then its area $S$ satisfies the inequality

$$
k_{n} S-\ell^{2} \leq 0,
$$

where $k_{n}$ is a positive constant depending on $n$ and the compact subset $K$.

Proof. The case $\kappa=0$ is due to S.Z. Shefel' $([20])$. Let $\kappa \neq 0$ and let $f$ be a saddle surface in $\mathbb{S}_{\kappa}^{n}$. Consider a continuous geodesic mapping $\varphi: \mathbb{S}_{\kappa}^{n} \rightarrow \mathbb{E}^{n}$. Such a geodesic mapping is the inclusion mapping $\varphi(x)=x$ from the BeltramiKlein model of $\mathbb{H}_{\kappa}^{n}$ to $\mathbb{E}^{n}$, if $\kappa<0$, and the central projection $\varphi\left(x_{1}, \ldots, x_{n+1}\right)=$ $\kappa^{-1 / 2}\left(x_{1} / x_{n+1}, \ldots, x_{n} / x_{n+1}\right)$, if $\kappa>0$. The surface $\varphi \circ f$ is a Euclidean saddle surface. It is not difficult to see that the restriction of $\varphi: \mathbb{S}_{\kappa}^{n} \rightarrow \mathbb{E}^{n}$ to a compact set is a bi-Lipschitz mapping. This observation has two consequences; there are positive 
constants $k_{1}, k_{2}$ such that for any curve $\gamma$ and any surface $f$ in the compact subset $K$ we have $k_{2} \ell(\gamma) \leq \ell(\varphi \circ \gamma) \leq k_{1} \ell(\gamma)$ and $k_{2}^{2} S(f) \leq S(\varphi \circ f) \leq k_{1}^{2} S(f)$, where $\ell$ denotes length and $S$ denotes the Lebesgue area. Therefore, since the Euclidean saddle surface $\varphi \circ f$ satisfies an isoperimetric inequality, so does the surface $f$ with coefficients depending on the compact subset $K$.

Theorem 21 enables us to strengthen Theorem 17 when $\mathfrak{R}_{\kappa}$ is a compact subset of $\mathbb{S}_{\kappa}^{n}$.

Theorem 22. If the lengths of the Jordan bounding curves of a family of saddle surfaces in a compact subset of $\mathbb{S}_{\kappa}^{n}$ are uniformly bounded, then the family being considered is a relatively compact subset of the space of surfaces.

The above theorem can be restated as follows.

Theorem 23. In order that a family of saddle surfaces in $\mathbb{S}_{\kappa}^{n}$ be relatively compact it is necessary and sufficient that the family of Jordan bounding curves of the given surfaces be relatively compact.

\section{ACKNOWLEDGMENT}

The author is indebted to Igor G. Nikolaev for the stimulating discussions on this work.

\section{REFERENCES}

[1] Aleksandrov A.D., Über eine Verallgemeinerung der Riemannschen Geometrie. Schr. Forchungsinst. Mat 1 (1957) 33-84 [German]. MR19:304h

[2] Aleksandrov A.D., Berestovskij V.N., Nikolaev I.G., Generalized Riemannian spaces, Russian Math. Surveys 41 (1986), 1-54. MR88e:53103

[3] Alexander S.B., Berg I.D., Bishop R.L., Geometric curvature bounds in Riemannian manifolds with boundary. Trans. Amer. Math. Soc. 339 (1993), 703-716. MR93m:53034

[4] Ballman W., Lectures on spaces of nonpositive curvature. DMV Seminar, Band 25, Birkhäuser, 1995. MR97a:53053

[5] Berestovskij V.N., Nikolaev I.G., Multidimensional generalized Riemannian spaces. Encyclopaedia of Math. Sciences, Vol. 70, Springer, Berlin, Heidelberg, New York, 1993, 184-242. MR 94i:53038

[6] Bridson M., Haefliger A., Metric spaces of nonpositive curvature. Springer, Berlin, Heidelberg, New York, 1999. MR2000k:53038

[7] Cesari L., Surface area. Princeton University Press, Princeton, New Jersey, 1956. MR 17:596b

[8] Courant R., Dirichlet's principle, conformal mapping, and minimal surfaces. Interscience Publishers, Inc., New York, Interscience Publishers LTD, London, 1977. MR 56:13103

[9] Jost J., Convex functionals and generalized harmonic maps into spaces of nonpositive curvature. Comment. Math. Helv. 70, No. 4 (1995), 659-673. MR.96j:58043

[10] Jost J., Nonpositive curvature: geometric and analytic aspects. Lectures in Mathematics ETH Zürich, Birkhäuser, 1997. MF $98 \mathrm{~g}: 53070$

[11] Kalikakis D., On the curvature of non-regular saddle surfaces in the hyperbolic and spherical three-space. Abstact and Applied Analysis, Vol. 7, No. 3 (2002), 113-123. MR2003a:53101

[12] Kalikakis D., A characterization of regular saddle surfaces in the hyperbolic and spherical three-space. Abstract and Applied Analysis, Vol. 7, No. 7 (2002), 349-355. MR.2003g:53011

[13] Korevaar N.J., Schoen, R.M., Sobolev spaces and harmonic maps for metric spaces targets. Comm. in Analysis and Geometry 1:4 (1993), 561-659. MR95b:58043

[14] McShane E.J., Parametrization of saddle surfaces with applications to the problem of Plateau. Trans. Amer. Math. Soc. 45, No. 3 (1933), 716-733.

[15] Nikolaev I.G., Solution of the Plateau problem in spaces of curvature not greater than $K$. Sib. Math. J. 20 (1979), 246-252. MR80k:58041

[16] Nikolaev I.G., Metric spaces of bounded curvature. Lecture notes, University of Illinois, Urbana, 1995 . 
[17] Osserman R., A survey of minimal surfaces. Dover Publication, Inc., New York, 1986. MR $87 \mathrm{j}: 53012$

[18] Reshetnyak Yu.G., Non-expanding mappings in a space of curvature not greater than $K$. Sib. Math. J. 9 (1968), 683-689.

[19] Shefel' S.Z., The intrinsic geometry of saddle surfaces. Sib. Mat. Zh. 5 (1964), 1382-1396 [Russian]. MR30:5232

[20] Shefel' S.Z., About saddle surfaces bounded by a rectifiable curve. Dokl. Akad. Nauk SSSR, Vol. 162, No 2 (1965), 294-296 [Russian]. MR31:3945

[21] Shefel' S.Z., Compactness conditions for a family of saddle surfaces. Sib. Math. J. 8 (1967), 528-535. MF 35:4826

Department of Mathematics, University of Crete, Irakleion, 714-09, Greece

E-mail address: kalikak@math.uoc.gr 